\title{
Stenotrophomonas maltophilia Measurement
}

National Cancer Institute

\section{Source}

National Cancer Institute. Stenotrophomonas maltophilia Measurement. NCI Thesaurus.

Code C139112.

The determination of the amount of Stenotrophomonas maltophilia present in a sample. 